This is a provisional PDF only. Copyedited and fully formatted version will be made available soon.

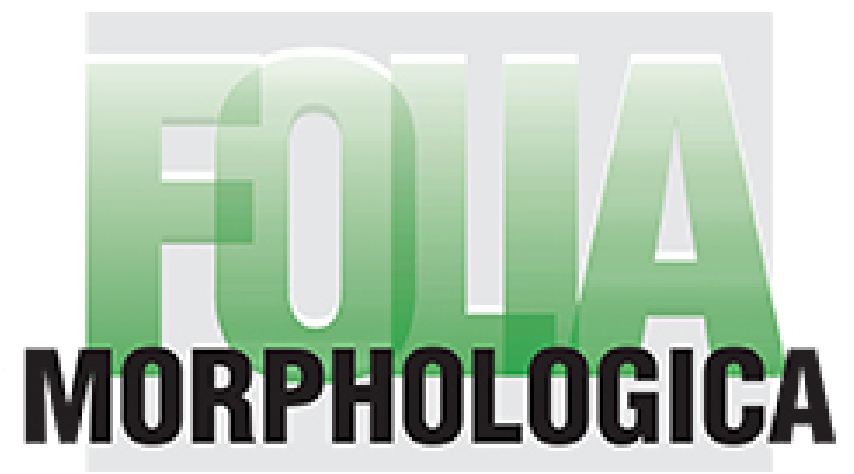

ISSN: 0015-5659

\title{
The spinal accessory nerve and its entry point into the posterior triangle of the neck
}

Authors: S. Ellis, C. Brassett, N. Glibbery, J. Cheema, S. Madenlidou

DOI: 10.5603/FM.a2022.0014

Article type: Original article

Submitted: $2021-11-03$

Accepted: $2022-02-02$

Published online: 2022-02-17

This article has been peer reviewed and published immediately upon acceptance.

It is an open access article, which means that it can be downloaded, printed, and distributed freely, provided the work is properly cited.

Articles in "Folia Morphologica" are listed in PubMed. 


\title{
The spinal accessory nerve and its entry point into the posterior triangle of the neck
}

S. Ellis et al., The spinal accessory nerve and its entry point into the posterior triangle of the neck

S. Ellis, C. Brassett, N. Glibbery, J. Cheema, S. Madenlidou

Human Anatomy Centre, Department of Physiology, Development and Neuroscience, University of Cambridge, Cambridge, United Kingdom

Address for correspondence: S. Ellis, BSc(Hons), MBChB, MRCS(ENT), Human Anatomy Centre, Department of Physiology, Development and Neuroscience, University of Cambridge, Downing Street, CB2 3EG Cambridge, United Kingdom, tel: 07967642 841, e-mail: sarah.ellis@doctors.org.uk

\begin{abstract}
The course of the spinal accessory nerve in the neck is long and superficial rendering it at high risk of injury during procedures performed in the posterior triangle. The majority of spinal accessory nerve injuries are iatrogenic in nature. This is associated with significant morbidity including reduction in shoulder movements, drooping of the shoulder, winging of the scapula and neuropathic pain. Knowledge of the nerve anatomy reduces the risk of intra-operative nerve injury. Traditional teaching describes the point of entry into the posterior triangle as the intersection between the upper and middle third of the posterior border of sternocleidomastoid. The aim of this study was to determine whether this is in fact the case and if so, whether this landmark can reliably be used to identify the spinal accessory nerve in order to improve patient outcomes.

The spinal accessory nerve was identified unilaterally in 26 cadavers. The total length of sternocleidomastoid was measured as well as the length along the posterior border from the inferior aspect of the mastoid process to the point at which the accessory nerve
\end{abstract}


enters the posterior triangle of the neck. These measurements were used to calculate the ratio of the entry point of the nerve into the posterior triangle along the length of the posterior border of sternocleidomastoid from its superior insertion point. The mean ratio was 0.35 with $95 \%$ confidence intervals of 0.33 to 0.36 .

Our findings confirm the traditional description of the entry point of the spinal accessory nerve into the posterior triangle of the neck. We describe a so-called 'safe zone' inferior to the midpoint of the posterior border of sternocleidomastoid within which the spinal accessory nerve is unlikely to be found, thereby reducing the risk of iatrogenic injury.

Key words: spinal accessory nerve, anatomy, posterior triangle, iatrogenic injury

\section{INTRODUCTION}

The accessory nerve is the $11^{\text {th }}$ cranial nerve and is derived from two roots; a spinal root and a cranial root[7]. The spinal accessory nerve exits the cranium via the jugular foramen, coursing through the neck, to provide motor supply to the sternocleidomastoid and trapezius muscles[7][1]. Its long and superficial course renders it at high risk of injury during procedures performed in the posterior triangle of the neck[1][10].

Most spinal accessory nerve injuries are iatrogenic in nature[6]. The literature demonstrates that it is one of the most commonly injured nerves during surgery across all specialties[20]. The anatomy of the nerve puts it at risk during otolaryngological surgery involving the lateral skull base, superior neck and posterior triangle[7][15]. This includes cervical lymph node biopsy, sebaceous cyst excision and neck dissection procedures[15]. Whilst the spinal accessory nerve is intentionally resected during a radical neck dissection, both modified-radical and selective neck dissections with nerve preservation are still associated with significant nerve morbidity[1,5,18]. Injury to the spinal accessory nerve results in the so-called 'Shoulder Syndrome'[16], characterised by paralysis of the trapezius muscle[10,13,17]. Clinical sequelae includes reduction in shoulder movements, drooping of the shoulder, winging of the scapula and neuropathic 
pain[10,13,17]. This morbidity, secondary to unintentional iatrogenic injury, has significant medicolegal repercussions as a source of malpractice litigation[15].

Detailed anatomical knowledge of the course of the spinal accessory nerve is fundamental to a reduction in the rate of iatrogenic injury. The nerve arises from the spinal nucleus at the levels C1-6 of the spinal cord[7,22]. These fibres merge to enter the posterior cranial fossa via the foramen magnum, after which the spinal root combines with the fibres of the cranial root to form a single common accessory nerve trunk[7,22]. The accessory nerve then exits the cranium via the jugular foramen, in close proximity to the internal jugular vein, before dividing again into a spinal and a cranial portion, with fibres from the latter running with the vagus nerve[7,22]. The spinal accessory nerve typically descends laterally to the internal jugular vein, then anterior to the transverse process of the atlas before passing medial to the styloid process, the stylohyoid and digastric muscles $[7,17,22]$. The nerve then enters or continues deep to the sternocleidomastoid muscle[17,22], before exiting from its posterior border to enter the posterior triangle of the neck, where it lies superficially $[7,17,22]$. The nerve then has a tortuous course as it continues to descend inferolaterally before exiting the posterior triangle by passing deep to the anterior border of trapezius[7,17,22].

Traditional anatomical teaching describes the point of entry of the spinal accessory nerve into the posterior triangle as the intersection between the superior onethird and inferior two-thirds of the length of the posterior border of sternocleidomastoid muscle[14,19]. The aim of this study was to determine the veracity of this statement and if so, whether this landmark can reliably be used to identify the spinal accessory nerve intra-operatively, in order to improve patient outcomes through avoidance of iatrogenic injury and associated morbidity.

\section{MATERIALS AND METHODS}

Unilateral neck dissections were performed on 26 embalmed cadavers at the Human Anatomy Centre, Department of Physiology, Development and Neuroscience, University of Cambridge. All donors had provided consent before decease for the use of their bodies for anatomical research, in compliance with the Human Tissue Act 2004. 
The side of the neck where the common carotid artery had not been used for vascular embalming was selected for dissection. The cadavers were placed in a supine position with a shoulder roll in order to extend the neck. The head was also rotated 45 degrees to the contralateral side. A standardised post-auricular skin incision was made. This was extended along the inferior aspect of the mandible, inferiorly at the midline of the anterior neck and along the clavicle laterally to the acromion. The skin flap was reflected laterally to expose the anterior triangle, the sternocleidomastoid muscle, the tip of the mastoid process and the posterior triangle of the neck. The sternocleidomastoid was cleared along its length to allow identification of insertion points, as was the anterior border of trapezius. Meticulous dissection of the posterior triangle allowed for identification of the accessory nerve and its entry and exit points along the sternocleidomastoid and trapezius muscles. The following measurements were taken:

— Length of posterior border of sternocleidomastoid (from the mastoid process superiorly to its attachment inferiorly onto the clavicle);

— Length along posterior border of sternocleidomastoid from its superior insertion point to the point at which the accessory nerve emerged to enter the posterior triangle; — Length of the anterior border of trapezius (from the clavicle inferiorly to its insertion at the superior nuchal line);

— Length along the anterior border of trapezius from the clavicle inferiorly to the point at which the accessory nerve exits the posterior triangle.

In order to reduce the risk of variability between measurements taken by differing researchers, the insertion points of sternocleidomastoid and trapezius were identified and marked by a single researcher using pins. String measurements were then taken. This was performed independently, and confirmed by two further researchers. Having obtained the above measurements, the mean length and standard deviations were calculated for each measurement as well as the ratio of the entry point of the accessory nerve into the posterior triangle along the length of the posterior border of 
sternocleidomastoid from its superior insertion point. The Pearson's correlation coefficient was also calculated in order to determine whether there was any significant relationship between this ratio and the height of the donor.

\section{RESULTS}

The spinal accessory nerve was identified unilaterally in 26 cadavers (14 male, 12 female). The median age was 83 years with a range of $67-100$ years. The average height and weight were $1.68 \mathrm{~m}$ and $63.0 \mathrm{~kg}$ respectively. Seventeen neck dissections were performed on the left and 9 on the right.

The mean length of the posterior border of sternocleidomastoid was 210mm +/23.2mm SD with a range of $171-260 \mathrm{~mm}$. The mean length from the superior insertion point to the entry point of the spinal accessory nerve into the posterior triangle, along the posterior border of sternocleidomastoid, was 73mm +/- 9.6mm SD.

The ratio of the entry point of the accessory nerve into the posterior triangle along the length of the posterior border of sternocleidomastoid from its superior insertion point ranged from 0.27 to 0.43 , with a mean of $0.35+/-0.04 \mathrm{SD}$ and $95 \%$ confidence intervals of 0.33 to 0.36 (Table 1 ).

Correlation studies were also performed to investigate the relationship between the height of the donor and the ratio of the entry point of the nerve into the posterior triangle from the superior insertion of sternocleidomastoid. The Pearson's correlation coefficient was $-0.29(\mathrm{p}<0.05)$. This weak, but significant correlation demonstrates that the entry point ratio does not alter with height. The mean entry point ratio was also 
calculated for male and female specimens; in males this was 0.34 and females it was 0.35 .

The mean exit point of the nerve from the posterior triangle was $39 \mathrm{~mm}$ along the anterior border of trapezius from the clavicle inferiorly.

\section{DISCUSSION}

The aims of this study were to determine if the entry point of the spinal accessory nerve into the posterior triangle of the neck conformed to traditional anatomical teaching, and whether this would be a reliable landmark for intra-operative identification of the nerve. Our findings corroborate traditional teaching which describes the point of entry of the spinal accessory nerve into the posterior triangle as the intersection between the superior one-third and inferior two-thirds of the length of the posterior border of sternocleidomastoid muscle (33\% of its length) $[14,19]$ In this study, the mean entry point of the nerve into the posterior triangle was 35\% of the total length of the posterior border of sternocleidomastoid from its superior insertion. With regards to whether this landmark should be used for intra-operative identification of the spinal accessory nerve, this study has demonstrated, with 95\% confidence intervals, that the ratio of the entry point of the accessory nerve into the posterior triangle along the border of sternocleidomastoid lies consistently between 33-36\% of its length from the superior insertion point, and that this is independent of height and sex.

There have been many attempts within the literature to describe the course of the spinal accessory nerve and to define its point of entry into the posterior triangle of the neck in relation to surrounding structures[8]. This includes distance from the clavicle[8,9], the mastoid process[3,4,8,11,12,23], the angle of the mandible[23], the greater auricular nerve[21] as well as, similar to this study, the distance along the posterior border of sternocleidomastoid from its superior insertion point[1,2,13,21,22]. 
This study found the ratio of the entry point of the accessory nerve into the posterior triangle along the length of the posterior border of sternocleidomastoid from its superior insertion point to be between 0.27 to 0.43 of the total length. Our findings suggest that iatrogenic injury to the spinal accessory nerve may be avoided in the majority of cases by limiting dissection inferior to half way along the posterior border of sternocleidomastoid. These findings are in keeping with those reported within the literature. Symes and Ellis(2005)[22] found that in a cadaveric study of 50 spinal accessory nerves, this ratio was between $0.28-0.44$ with a mean entry point of 0.36 . Similarly, Abakay et al. (2020)[1] found that this ratio was between 0.25-0.40 in 87 out of 100 specimens. Soo et al. (1986) reported that in 31 of 32 cadaveric specimens the spinal accessory nerve entered the posterior triangle within the superior half of the border of sternocleidomastoid with the nerve only entering outside of this in area $3 \%$ of cases[21]. As such, we can conclude that the majority of iatrogenic injuries to the spinal accessory nerve may be avoided by limiting dissection to the inferior half of sternocleidomastoid and cautious dissection superiorly.

There are methodological limitations to this study including a small sample size of only 26 nerves and the potential for variation in the points between which measurements were taken, for example, when identifying the insertion point of sternocleidomastoid and trapezius muscles. In an attempt to overcome inter-rater variability, a single researcher identified these points with pins for all specimens and two researchers subsequently took all the measurements which were then compared and repeated. However, in order to replicate the clinical environment, we ensured standard positioning with shoulder roll and neck rotated 45 degrees to the contralateral side.

\section{CONCLUSIONS}


The principal aim of this study was to determine whether the entry point of the spinal accessory nerve into the posterior triangle is, as described in traditional anatomical teaching, at the intersection between the superior one-third and inferior twothirds of the length of the posterior border of sternocleidomastoid muscle[14,19]; our findings do confirm this description. In addition, we describe a so-called 'safe zone' of the posterior triangle of the neck, inferior to the midpoint of the posterior border of sternocleidomastoid, within which the spinal accessory nerve is unlikely to be found, thereby reducing the risk of iatrogenic injury.

\section{Acknowledgements}

Donors and their families without whom this study would not have been possible as well as the Dissection Room technical staff at the Human Anatomy Centre, Department of Physiology, Development and Neuroscience, University of Cambridge.

\section{Conflict of interest: None declared}

\section{REFERENCES}

[1] Abakay MA, Güneş S, Küçük C, et al. Accessory Nerve Anatomy in Anterior and Posterior Cervical Triangle: A Fresh Cadaveric Study. Turk Arch Otorhinolaryngol. 2020 Sep;58(3):149-154. doi: 10.5152/tao.2020.5263.

[2] Amuti T, Butt F, Otieno B, et al. The relation of the extracranial spinal accessory nerve to the sternocleidomastoid muscle and the internal jugular vein. Craniomaxillofacial trauma \& reconstruction. 2019; 12(2), pp.108-111.

[3] Canella C, Demondion X, Abreu E, et al. Anatomical study of spinal accessory nerve using ultrasonography. European journal of radiology, 2013; 82(1), pp.56-61.

[4] Dailiana ZH, Mehdian H, Gilbert A. Surgical Anatomy of Spinal Accessory Nerve: Is Trapezius Functional Deficit Inevitable after Division of the Nerve? Journal of Hand Surgery. 2001;26(2):137-141. doi:10.1054/jhsb.2000.0487

[5] Goldstein David P, Ringash J, Bissada E, et al. Scoping Review of the literature on shoulder impingements and disability after neck dissection. Journal of the sciences and specialties of the head and neck. 2014: 36,2: 299-308. doi.org:10.1002/hed.23243.

[6] Grossman JA, Ruchelsman DE, Schwarzkopf R. Iatrogenic spinal accessory nerve injury in children. Journal of pediatric surgery. 2008 Sep 1;43(9):1732-5. 
[7] Johal J, Iwanaga J, Tubbs K, et al, The Accessory Nerve: A Comprehensive Review of its Anatomy, Development, Variations, Landmarks and Clinical Considerations. Anat. Rec. 2019; 302: 620-629, doi.org/10.1002/ar.23823.

[8] Khavanin N, Carl HM, Yang R, et al. Surgical "Safe Zone": rapid anatomical identification of the lesser occipital nerve. Journal of reconstructive microsurgery, 2019; 35(05), pp.341-345.

[9] Kierner AC, Zelenka I, Heller S, et al. Surgical anatomy of the spinal accessory nerve and the trapezius branches of the cervical plexus. Archives of Surgery, 2000; 135(12), pp.1428-1431.

[10] London J, London N, Kay SP. Iatrogenic accessory nerve injury. Annals of the Royal College of Surgeons of England. 1996 Mar 78(2):146.

[11] Lu J, Haman SP and Ebraheim, N.A. Vulnerability of the spinal accessory nerve in the posterior triangle of the neck: a cadaveric study. The anatomical record, special issue: The cranial nerves: morphology and clinical relevance. 2002: 302(4) pp620-629 doi.org/10.1002/ar23823.

[12] Lloyd, S., 2007. Accessory nerve: anatomy and surgical identification. The Journal of Laryngology \& Otology, 121(12), pp.1118-1125.

[13] Mirjalili, S.A., Muirhead, J.C. and Stringer, M.D. Ultrasound visualization of the spinal accessory nerve in vivo. Journal of surgical research, 2012 175(1), pp.e11-e16

[14] Moore KL, Dalley AF. Clinically orientated anatomy, $3^{\text {rd }}$ edition. Lippincott, Williams and Wilkins, Philadelphia 1992.

[15] Morris LGT, Ziff DJS, DeLacure MD. Malpractice Litigation After Surgical Injury of the Spinal Accessory Nerve: An Evidence-Based Analysis. Arch Otolaryngol Head Neck Surg. 2008;134(1):102107. doi:10.1001/archotol.134.1.102.

[16] Nahum AM, Mullally W, Marmor L. A Syndrome Resulting from Radical Neck Dissection. Arch Otolaryngol. 1961;74(4):424-428. doi:10.1001/archotol.1961.00740030433011

[17] Overland J, Hodge J, Breik O, et al. Surgical anatomy of the spinal accessory nerve: Review of the literature and case report of a rare anatomical variant. 2016 The Journal of Laryngology \& Otology, 130(10), 969-972. doi:10.1017/S0022215116008148

[18] Popovski V, Benedetti A, Popovic-Monevska D, et al. Spinal accessory nerve preservation in modified neck dissections: surgical and functional outcomes. Acta Otorhinolaryngol Ital. 2017 Oct;37(5):368-374. doi: 10.14639/0392-100X-844. PMID: 29165431; PMCID: PMC5720864.

[19] Romanes GF, Cunningham DJ. Cunningham's textbook of anatomy, $12^{\text {th }}$ edition. Oxford University Press, Oxford 1981.

[20] Sharp E, Roberts M, Żurada-Zielińska A, et al. The most commonly injured nerves at surgery: A comprehensive review. Clinical Anatomy. 2021: 34(2):244-62.

[22] Symes, A., Ellis, H. Variations in the surface anatomy of the spinal accessory nerve in the posterior triangle. Surg Radiol Anat 2005 27, 404-408. https://doi.org/10.1007/s00276-005-0004-9

[21] Soo KC, Hamlyn PJ, Pegington J, et al. Anatomy of the accessory nerve and its cervical contributions in the neck. Head \& neck surgery. 1986; 9(2), pp.111-115.

[23] Tubbs, R.S., Salter, E.G., Wellons, J.C., et al. Superficial landmarks for the spinal accessory nerve within the posterior cervical triangle. Journal of Neurosurgery: Spine. 2005; 3(5), pp.375-378. 
Table 1. Measurements taken and entry point of SAN into the posterior triangle

\begin{tabular}{|l|c|c|c|}
\hline Measurement & Mean & $\begin{array}{c}\text { Standard } \\
\text { Deviation (mm) }\end{array}$ & Range (mm) \\
\hline Length posterior border of SCM* & $210 \mathrm{~mm}$ & 23.3 & $171-260$ \\
\hline $\begin{array}{l}\text { Length from superior insertion point } \\
\text { SCM* to entry point of SAN** into } \\
\text { posterior triangle }\end{array}$ & $73 \mathrm{~mm}$ & 9.6 & $51-90$ \\
\hline $\begin{array}{l}\text { Ratio of entry point SAN** into posterior } \\
\text { triangle along length of posterior border } \\
\text { SCM }^{*}\end{array}$ & 0.35 & 0.04 & $0.27-0.43$ \\
\hline
\end{tabular}

*SCM $=$ Sternocleidomastoid **SAN $=$ Spinal Accessory Nerve 